\title{
Image-guided device therapy: An opportunity for personalized medicine
}

\author{
Prem Soman, MD, PhD, ${ }^{\mathrm{a}}$ and Sandeep K. Jain, $\mathrm{MD}^{\mathrm{a}}$ \\ a Division of Cardiology and The Heart and Vascular Institute, University of Pittsburgh Medical \\ Center, Pittsburgh, PA
}

Received Jul 30, 2020; accepted Jul 30, 2020

doi: $10.1007 / \mathrm{s} 12350-020-02327-6$

\section{See related article, pp. 1153-1161}

The implantation of the first internal cardiac pacemaker in 1958 heralded the era of implantable cardiac devices $^{1}$. The subsequent evolution of the cardiac pacemaker through stages of progressive sophistication reflects still extant efforts to mimic the physiology of native conduction. The right ventricular (RV) apex has traditionally been used as the preferred site of pacing due to the relative ease of access and the favorable logistics of securing the pacemaker lead in the trabeculae. Single-chamber RV pacing was followed by dualchamber pacing to maintain atrioventricular synchrony. However, studies have demonstrated deleterious effects of RV apical pacing on left ventricular (LV) function due to mechanical dyssynchrony resulting from the spread of the activation wavefront through the myocardium rather than specialized conduction tissue ${ }^{2}$. The iatrogenic wide-QRS left bundle branch block (LBBB) produced by RV apical pacing results in an extreme degree of LV intraventricular dyssynchrony. Progressive deterioration of LV systolic function after RV apical pacing is a well-recognized phenomenon and has spurred the practice of pre-emptive biventricular pacing in patients with LV systolic dysfunction who are expected to be dependent on a substantial burden of paced beats. Quite remarkably, however, despite evidence of betterpreserved LV systolic function ${ }^{3}$ there have been relatively few efforts to substantiate the notion of less

Reprint requests: Prem Soman, MD, PhD, Division of Cardiology and The Heart and Vascular Institute, University of Pittsburgh Medical Center, A-429 Scaife Hall, 200 Lothrop Street, Pittsburgh, PA 15213; premsoman@usa.net

J Nucl Cardiol 2021;28:1162-4.

$1071-3581 / \$ 34.00$

Copyright (c) 2020 American Society of Nuclear Cardiology. mechanical dyssynchrony with biventricular compared to RV pacing. Continuing efforts to optimize the physiology of cardiac pacing have resulted in proposals for RV septal pacing and His-bundle pacing. ${ }^{4,5}$ The latter approach, although theoretically the most physiological, is fraught with the technical challenges of lead stability and increasing pacing thresholds over time. ${ }^{6}$ More recently, left bundle branch (LBB) pacing has been proposed $^{7}$ as an alternative to His-bundle pacing with the hope of overcoming some of these shortcomings with potential improved long-term stability and thresholds allowing maximal battery life. Unique downsides to LBB pacing are the possibility of septal perforation and potential coronary injury in addition to injury to the HisPurkinje system with repositioning, all of which need to be evaluated in larger studies.

The use of radionuclide myocardial perfusion imaging to measure LV synchrony is now well-established ${ }^{8}$. This approach leverages the partial volume effect, which results in a relatively linear relationship between regional myocardial thickening and brightening within the range of myocardial thicknesses typically encountered. Thus, a time-intensity curve of the myocardium is essentially its thickening curve, albeit with a poor resolution due to the 8- or 16-bin gating. Fourier transformation results in a continuous thickening curve, from which the phase of the onset (or peak) of myocardial contraction can be determined. Regional time-intensity curves are used to determine the phase of the onset (or peak) of contraction of myocardial segments. It follows logically that synchronously contracting myocardial segments will have a similar phase of onset (or peak) of contraction. The phase histogram bandwidth (PHB) and phase standard deviation (PSD) are established measures of LV intraventricular dyssynchrony.

In this issue of the Journal, Qian, and colleagues from the Nanjing Medical University report on an interesting and pragmatic study on the important clinical topic of identifying LBB capture ${ }^{9}$. They postulate that 
the metric of LV activation time (LVAT), which is analogous to the intrinsicoid deflection of the QRS complex, may be useful to determine if LBB capture has occurred in cases where an LBB signal cannot be obtained. They use SPECT-derived dyssynchrony indices as a surrogate of LBB capture based on the premise that $\mathrm{LV}$ activation through the left bundle will produce less LV dyssynchrony compared to LV septal pacing. Patients were classified based on the mean LVAT for the study population of $76 \mathrm{~ms}$ (which serendipitously, corresponds to the literature on LBB pacing). They report that the combination of a high LVAT and an inability to record a measurable LBB signal was associated with the worst LV synchrony (Figure 3 in the article). They report that patients with an LVAT $<76 \mathrm{~ms}$ had the smallest QRS duration. This, of course, is somewhat of a self-fulfilling prophecy. The LVAT was correlated with SPECT-derived mechanical synchrony, although with values of $r$ that were only 0.27 and 0.31 for PHB and PSB, respectively. They also show that when the LVAT is less than $76 \mathrm{~ms}$, the measured LV synchrony was similar (no more than $50 \%$ different) to that obtained during right atrial pacing in patients with normal atrioventricular conduction.

There are several limitations to this study, which should, therefore, be considered no more than hypothesis-generating. The cut off value of LVAT was derived and validated in the same group, and its measurement error was not defined. The parameter of a less than 50\% difference in synchrony for comparison with right atrial pacing was arbitrary. The $r$ values for correlation were modest at best and clinical outcomes were not measured. The technique as described is also limited in that the surrogate to dyssynchrony (LVAT) is apparent only after lead deployment. A physiologic guide/predictor before lead deployment to minimize repositioning would be ideal.

Nevertheless, the investigators creatively leverage the strengths of imaging to calibrate the settings of a cardiac device to optimize physiology. Such an approach offers unique opportunities for personalized medicine. Cardiac device therapy is expensive. Response rates to CRT and implantable cardioverterdefibrillator therapy are often suboptimal when patients are selected based on broad criteria such as an ejection fraction cut-off. In the field of cardiac resynchronization therapy (CRT), the delivery of the LV lead to a myocardial segment that is delayed in its activation, and not scarred has been shown to improve patient outcome $^{10,11}$. Specific conduction abnormalities likely result in unique patterns of dyssynchrony. For example, an LBBB predictably results in a septal to lateral delay in activation, while RBBB and non-specific intraventricular conduction abnormalities result in less predictable patterns (unpublished data from our lab). Thus, imaging offers the opportunity to uniquely tailor device settings to an individual patient's pathophysiology. In this era of cost-consciousness in which our healthcare delivery paradigms are being shifted from fee-for-service to value-based care, imaging could have an important role to play in individualizing therapy.

\section{Disclosures}

Dr. Soman has no relevant disclosure. Dr. Jain has research grants from Boston Scientific and Medtronic.

\section{References}

1. Aquilina O. A brief history of cardiac pacing. Images Paediatr Cardiol. 2006;8:17-81.

2. Tantengco MVT, Thomas RL, Karpawich P. Left ventricular dysfunction after long-term right ventricular apical pacing in the young. J Am Coll Cardiol. 2001;37:203-2100.

3. Curtis AB. Biventricular pacing for atrioventricular block and systolic dysfunction. N Engl J Med. 2013;369:579. https://doi.org/ 10.1056/NEJMc1306998.

4. Deshmukh P, Casavant DA, Romanyshyn M, Anderson K. Permanent, Direct His-Bundle Pacing. Circulation. 2000;101:869-77.

5. Hussain MA, Furuya-Kanamori L, Kaye G, Clark J, Doi SAR. The effect of right ventricular apical and non-apical pacing on the short- and long-term changes in left ventricular ejection fraction: A systematic review and meta-analysis of randomized controlled trials. PACE Pacing Clin. Electrophysiol. 2015;38:1121-36.

6. Barakat AF, Inashvili A, Alkukhun L, Shalaby AA, Wang NC, Bhonsale A, Wann D, Gardner M, Khan M, Bidani S, Skowronski J, Jain S, Voigt A, Bazaz R, Estes NAM III, Saba S, Kancharla K. Use trends and adverse reports of Select Secure 3830 lead implantations in the United States: implications for his bundle pacing. Circ Arrhythmia Electrophysiol. 2020;13:704-6.

7. Huang W, Su L, Wu S, Xu L, Xiao F, Zhou X, Ellenbogen KA. A novel pacing strategy with low and stable output: pacing the left bundle branch immediately beyond the conduction block. Can J Cardiol. 2017;33:1736.e1-3.

8. Chen J, Garcia EV, Bax JJ, Iskandrian AE, Borges-Neto S, Soman P. SPECT myocardial perfusion imaging for the assessment of left ventricular mechanical dyssynchrony. J Nucl Cardiol. 2011;18:685-94.

9. Barakat AF, Inashvili A, Alkukhun L, Shalaby AA, Wang NC, Bhonsale A, Wann D, Gardner M, Khan M, Bidani S, Skowronski J, Jain S, Voigt A, Bazaz R, Estes Iii NAM, Saba S, Kancharla K. Use trends and adverse reports of SelectSecure 3830 lead implantations in the united states: implications for his bundle pacing. Circ Arrhythm Electrophysiol. 2020;13:704-5.

10. Friehling M, Chen J, Saba S, Bazaz R, Schwartzman D, Adelstein EC, Garcia E, Follansbee W, Soman P. A prospective pilot study to evaluate the relationship between acute change in left ventric- 
ular synchrony after cardiac resynchronization therapy and patient outcome using a single-injection gated SPECT protocol. Circ Cardiovasc Imaging. 2011;4:532-9.

11. Saba S, Marek J, Schwartzman D, Jain S, Adelstein E, White P, Oyenuga OA, Onishi T, Soman P, Gorcsan J. Echocardiographyguided left ventricular lead placement for cardiac resynchronization therapy results of the speckle tracking assisted resynchronization therapy for electrode region trial. Circ Heart Fail. 2013;6:427-34.

Publisher's Note Springer Nature remains neutral with regard to jurisdictional claims in published maps and institutional affiliations. 